\title{
NanoJapan: international research experience for undergraduates program: fostering U.S.-Japan research collaborations in terahertz science and technology of nanostructures
}

Sarah Phillips, Cheryl Matherly, Junichiro Kono

Sarah R. Phillips, Cheryl A. Matherly, Junichiro Kono, "NanoJapan: international research experience for undergraduates program: fostering U.S.Japan research collaborations in terahertz science and technology of nanostructures," Proc. SPIE 9188, Optics Education and Outreach III, 918805 (15 September 2014); doi: 10.1117/12.2060166

EviE Event: SPIE Optical Engineering + Applications, 2014, San Diego, California, United States 


\title{
NanoJapan--International research experience for undergraduates program: Fostering U.S.-Japan research collaborations in terahertz science and technology of nanostructures
}

\author{
Sarah R. Phillips*a , Cheryl A. Matherly ${ }^{\mathrm{b}}$, and Junichiro Kono ${ }^{\mathrm{a}}$
}

${ }^{a}$ Department of Electrical and Computer Engineering, Rice University, 6100 Main St., Houston, TX 77005, USA; ${ }^{b}$ Center for Global Education, University of Tulsa, 800 South Tucker Dr., Tulsa, OK 74104, USA

\begin{abstract}
The international nature of science and engineering research demands that students have the skillsets necessary to collaborate internationally. However, limited options exist for science and engineering undergraduates who want to pursue research abroad. The NanoJapan International Research Experience for Undergraduates Program is an innovative response to this need. Developed to foster research and international engagement among young undergraduate students, it is funded by a National Science Foundation Partnerships for International Research and Education (PIRE) grant. Each summer, NanoJapan sends 12 U.S. students to Japan to conduct research internships with world leaders in terahertz $(\mathrm{THz})$ spectroscopy, nanophotonics, and ultrafast optics. The students participate in cutting-edge research projects managed within the framework of the U.S-Japan NSF-PIRE collaboration. One of our focus topics is THz science and technology of nanosystems (or 'TeraNano'), which investigates the physics and applications of THz dynamics of carriers and phonons in nanostructures and nanomaterials. In this article, we will introduce the program model, with specific emphasis on designing high-quality international student research experiences. We will specifically address the program curriculum that introduces students to $\mathrm{THz}$ research, Japanese language, and intercultural communications, in preparation for work in their labs. Ultimately, the program aims to increase the number of U.S. students who choose to pursue graduate study in this field, while cultivating a generation of globally aware engineers and scientists who are prepared for international research collaboration.
\end{abstract}

Keywords: terahertz science and technology, nanostructures and nanomaterials, global education, undergraduate research

\section{INTRODUCTION}

Science and engineering (S\&E) research is increasingly characterized by international collaboration, but few U.S. undergraduates in these fields participate in international experiences. In 2011/2012, the most current academic year for which data is available, only $3.9 \%$ of the students who studied abroad majored in engineering, $8.6 \%$ in the physical and life sciences, and $1.7 \%$ in mathematics and computer science. ${ }^{1}$ The small number of U.S. S\&E students who pursue an international experience is particularly concerning given the importance being placed on international research collaborations in S\&E by national agencies and professional organizations. In its 2006-2011 fiscal year strategic report, the National Science Foundation (NSF) identified as a key performance goal the need to "keep the United States at the frontiers of knowledge by increasing international partnerships and collaborations." The strategic plan went on to say, "As S\&E expertise and infrastructure advance across the globe, it is expected that the United States will increasingly benefit from international collaborations and a globally engaged workforce leading to transformational S\&E breakthroughs."

The need for intercultural competency and maturity is particularly important for S\&E students, who are increasingly working alongside international students at both the undergraduate and graduate levels. While the number of international graduate students in S\&E fields remained stable from 2011 to 2012, the number of international students pursuing undergraduate degree study in S\&E fields in the U.S. increased by $9.1 \%$ in engineering and $4.8 \%$ in the physical and life sciences. ${ }^{3}$ Thus, not only is the development of intercultural maturity and competencies vital to student's future success as graduate students and researchers, it can also be translated into their day-to-day experience as students at campuses nationwide, where students will increasingly find themselves in classroom, living, and research labs with students from around the world.

Optics Education and Outreach III, edited by G. Groot Gregory, Proc. of SPIE Vol. 9188 ,

918805 @ 2014 SPIE $\cdot$ CCC code: 0277-786X/14/\$18 · doi: 10.1117/12.2060166 
The NanoJapan Program was developed to address this need by attracting undergraduates to the emerging areas of nanoscience. Funded by an NSF Partnerships for International Research and Education (NSF-PIRE) grant, each summer we send 12 U.S. students to collaborating labs in Japan, world leaders in terahertz (THz) spectroscopy, nanophotonics, and ultrafast optics. The students participate in cutting-edge research projects managed within the framework of the U.S-Japan PIRE collaboration. One of our focus topics is THz science and technology of nanosystems (or 'TeraNano'), which investigates the physics and applications of THz phenomena in nanostructures and nanomaterials. NanoJapan tightly integrates the international experience with students' academic programs by providing hands-on opportunities to acquire technical skills and knowledge associated with cutting-edge nanoscience and optics research projects. The program aims to increase the number of U.S. students who pursue graduate study in optics and photonics with nanosystems and cultivate a generation of globally aware scientists and engineers who are prepared for international research collaboration.

\section{PROGRAM DESIGN}

NanoJapan, like all PIRE-funded projects, is characterized by a tight integration between the research and education programs. The education program is informed by Parkinson's characteristics for a 'globally competent engineer,' in that it encourages students to appreciate other cultures, develop proficiency in working in cross-cultural teams, communicate across cultures, practice engineering in a global environment, and evaluate ethical issues arising from cultural differences. ${ }^{4}$ The program model is described below.

\section{Participants}

The NanoJapan Program recruits freshmen and sophomore science and engineering (S\&E) students from universities nationwide. To be eligible to apply, students must be a U.S. citizen or permanent resident. No prior research or Japanese language experience is required, but students must be able to express a demonstrated interest in science research and Japan. Students submit an online application, and our team conducts phone interviews with finalists in order to select the 12 student participants in each year. The NanoJapan Program is highly competitive, and in the 2014 program year alone, NanoJapan received 160 applications; a selection rate of just 7.5\%.

Since 2006, 130 students have participated, representing 42 different universities including three community colleges and minority-serving institutions such as Morehouse College. Of the students who have graduated, 75 percent are pursuing or have received a graduate degree in a science, technology, engineering, or mathematics (STEM) field. The NanoJapan Program has a strong track record of recruiting underrepresented S\&E students, with $13.8 \%$ of participants representing diverse ethnic groups in S\&E fields. Female students represent 33.8\% of NanoJapan participants overall. The representation of women within the program is particularly impressive given that NSF data shows that in 2010, the last year for which data is available, conferred bachelor's degrees for female undergraduate students represented just $16.98 \%$ of all engineering degrees conferred and $20.41 \%$ of physics degrees. ${ }^{5}$

The decision to target students early in their college career was a response to the low rates of participation in study abroad by S\&E majors. The program is designed to be a 'catalyst': the goal is to introduce students to research and international study when they are freshmen and sophomores so that they may choose to continue language study, seek other domestic or international research experiences for undergraduates, or study abroad again, based on what they learn from the NanoJapan Program. For many students, the program represents their first experience conducting research and first exposure to Japan. Many of the students are attracted first to the NanoJapan Program because it is a scientific research program. Most have also indicated that they consider NanoJapan their best alternative to a traditional study abroad program; which they would be unlikely to pursue since it would require them to miss a regular semester of courses.

\section{Pre-Departure Orientation at Rice University (Mid-May, 1 day)}

Before leaving for Japan, students participate in a one-day orientation in which they are introduced to approaches to thinking about cultural differences. Throughout the summer, students engage in activities that are prompt intentional reflections on differences between scientific research in the U.S. and Japan. Facilitated reflection is widely understood a best practice for encouraging cross-cultural learning during study abroad. ${ }^{6}$ The team has used a variety of techniques, but most recently used the Cultural Detectives program, in which students compare cultural values for the U.S. and Japan and learn how to evaluate cross-cultural miscommunications. The pre-departure orientation also incorporates health, safety, and risk workshops in keeping with best practices for short-term study abroad programs. ${ }^{7}$ 


\section{Japanese Language and Culture Orientation in Tokyo (Mid-May to Early June, 3 weeks)}

Students complete a three-week orientation program in Tokyo that introduces them to TeraNano research and the competencies required to work successfully in the global science community. Since most NanoJapan participants have not had previous experience in Japan, the students complete 45 hours of intensive Japanese language instruction. Beginning students are taught by language school faculty, and intermediate/advanced language students are taught by the PIRE Japanese Language Director, Prof. Mitsuaki Shimojo, based on the Technical Japanese course he has developed at the University at Buffalo. Additionally, students participate in a colloquium on the culture and society of Japan, taught by local Japanese faculty. Finally, they participate in a seminar series that introduces them to TeraNano research that is led by PI Kono of our NSF-PIRE grant and his co-PIs from collaborating universities in the U.S. (Prof. Jonathan Bird at the University at Buffalo and Prof. Christopher Stanton at the University of Florida). The seminar series also includes guest lectures from leading Japanese $\mathrm{THz}$ researchers, lab visits, and company visits. The goal of the orientation program is to provide participants with the basic language and intercultural communication skills that give them a firm foundation upon which they can build during their research internships.

International Research Experience (IRE) in Nanotechnology (June - July, 8 weeks)

\section{TeraNano PIRE Japanese Partners}

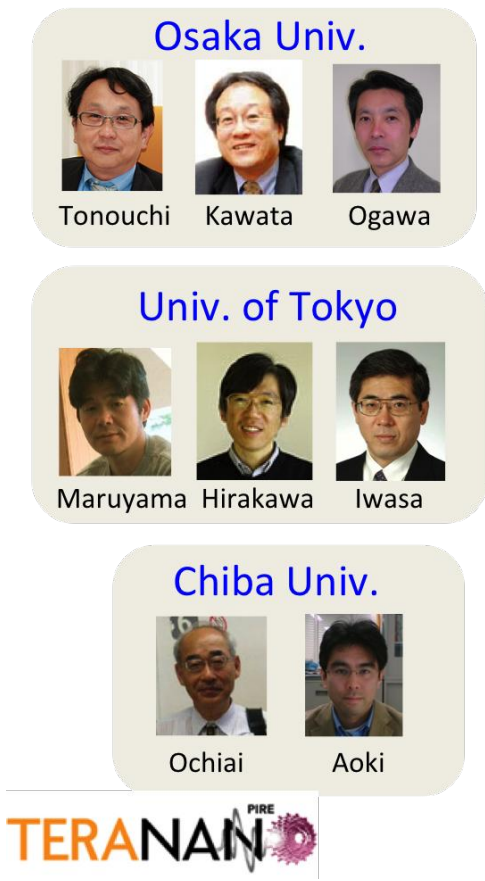

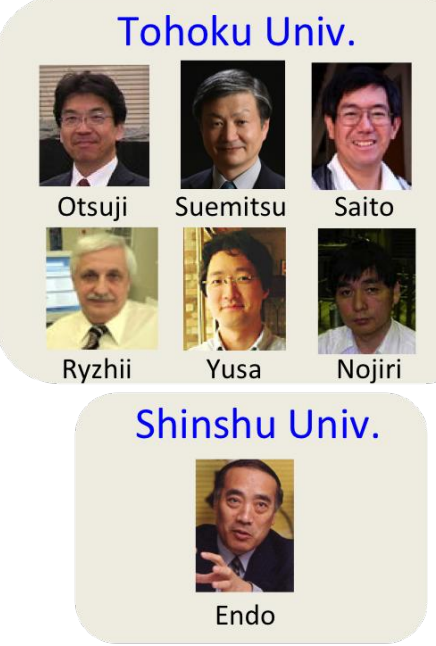

Tokyo Inst. of Technol.

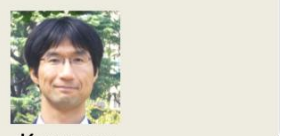

Kawano

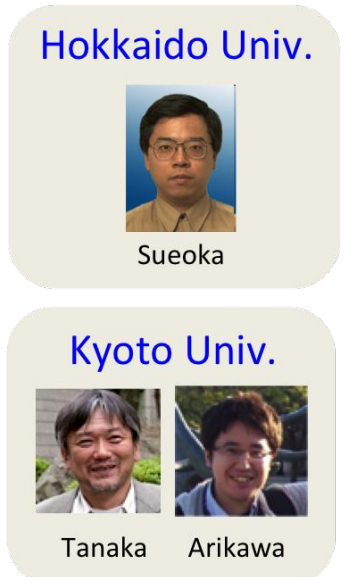

Okinawa Inst. of

Sci. \& Technol.

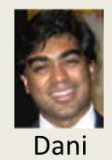

Figure 1. TeraNano PIRE research collaborators and NanoJapan host advisors in Japan include leading terahertz and nanoscience researchers at Osaka University, the University of Tokyo, Chiba University, Tohoku University, Shinshu University, Tokyo Institute of Technology, Hokkaido University, Kyoto University, and the Okinawa Institute of Science \& Technology Graduate University.

Following the completion of the program in Tokyo, students are assigned to leading TeraNano research labs throughout Japan for eight-week summer internships, where they conduct hands-on research projects. The U.S. research team, in collaboration with the Japanese host laboratories, develops student research projects prior to arrival at the research host lab. Each NanoJapan student, under the co-advisement of both a Japanese and U.S. PIRE research professor, is assigned to a single project; while there may be other NanoJapan students in the same university or host city, she/he will be the only NanoJapan student working on that particular project. The international dimension of their projects is heighted as each NanoJapan student is mentored on a day-to-day basis by a Japanese graduate student or post-doctoral mentor, closely mirroring the experience of being a graduate student working on an international research collaboration. 
The primary language used in most research labs is English, though levels of English spoken by members of the host lab may vary. All Japanese PIRE research professors speak English, and the Japanese graduate student mentors assigned to work with the NanoJapan students must also be able to speak English with the U.S. students. Japanese lab members benefit from having the U.S. student in their group as they are able to practice and improve upon their conversational English skills, important to future international collaborations that they may be a part of. Though most students will primarily communicate about their research project in English, NanoJapan students also must draw upon the Japanese language skills attained during the orientation program to effectively communicate with all members of their lab, and their local community, and develop strategies to overcome language barriers with their research colleagues. Students have the option of continuing formal Japanese language study via any language classes that may be offered at their host university, though most chose to pursue one-on-one tutoring or language exchanges with their Japanese research group members, or self-study supplemented by online resources provided by the NanoJapan Program.

\section{Mid-Program Meeting \& Research Host Lab Visits (Early July, 3 days)}

Held as a three-day meeting in the middle of the eight-week IRE, this meeting brings together all NanoJapan students along with U.S. PIRE faculty advisors to debrief on the research and cultural experience in Japan to date. Through facilitated discussion, students are able to share with their fellow program participants some of the challenges, frustrations, successes, and best practices they have encountered as an American student doing research and living in Japan. In conjunction with this meeting, students also participate in a cultural workshop introducing them to the culture of the city or region in which the meeting is held.

In 2013 and 2014, the Mid-Program Meeting was held in Okinawa and was hosted by the Okinawa Institute of Science and Technology Graduate University (OIST), an interdisciplinary graduate school offering a 5-year Ph.D. program in science. Over half of the faculty and students at OIST are recruited from outside Japan, and all education and research is conducted entirely in English. NanoJapan students received an introduction to this new model of graduate education in Japan and met with leading $\mathrm{THz}$ and nanoscience researchers. Our collaboration with OIST provides NanoJapan students with a better understanding of graduate education and research in Japan and some of the new models being developed by academic institutions and government initiatives to foster stronger international engagement and collaboration, particularly in emerging fields such as THz science and technology of nanosystems.

\section{NanoJapan Research Symposium in Tokyo (Early August, 1 day)}

Upon the conclusion of the IRE, students return to Tokyo for a one-day research symposium that all Japanese PIRE professors and research mentors are invited to attend. The symposium highlights U.S. and Japanese student collaborations in TeraNano research and provides an opportunity for NanoJapan students to jointly present on their research projects alongside their Japanese mentors. In 2014, the topic of this workshop was expanded to highlight "Preparing Global Researchers: Facilitating Effective U.S.-Japanese Student Research Collaboration" and included guest talks by representative of Japanese funding agencies on national initiatives in Japan to expand opportunities for international collaborations and engagement for Japanese students.

\section{Re-Entry and Career Education Program (Early August, 2 days)}

Upon the conclusion of the research symposium in Tokyo, NanoJapan students return to Rice University for a re-entry and career education program. The re-entry program is specifically designed for the needs of young career scientists. In addition to examining issues associated with re-entry to the U.S., students participate in a Rice Center for Engineering Leadership sponsored workshop on poster presentation skills and receive one-on-one coaching from trained presentations coaches. The re-entry program also includes workshops on marketing your international experience, graduate study in S\&E fields, international fellowships and scholarships, further opportunities for experiences abroad, and career development and training activities including resume review and mock interview sessions. Combined, these activities help students evaluate their experience in Japan in preparation for graduate school and identify next academic, international, or other experiential programs that can advance their personal and professional goals.

\section{Rice Quantum Institute Summer Research Colloquium (Early August, 1 day)}

The capstone experience of the NanoJapan Program is participation in the Annual Rice Quantum Institute Summer Research Colloquium, where NanoJapan students present posters on their research experience in Japan along with other U.S. students who have completed quantum-related summer research experiences at Rice University. The Colloquium provides an opportunity for student researchers to make professional presentations and prepare a 'product' at the conclusion of their summer internships. 


\section{Other Program Logistics}

The NSF-PIRE grant provides funding for international airfare, housing during the three-week orientation in Tokyo, 45 hours of intensive Japanese language costs, domestic travel to the Mid-Program Meeting in Okinawa, and workshop and seminar costs for instructors and guest speakers. Each NanoJapan student receives a stipend of $\$ 4,000$ to be used for their daily meal costs and housing during the research internship period. Housing during the research internship is arranged on behalf of the student by their research host lab and is typically in university dorms or housing for short-term, visiting researchers. Students are individually responsible for domestic airfare to/from Houston, a program fee, passports/immunizations, Japanese cell phone costs, and the cost of personal travel/sightseeing while in Japan.

NanoJapan is a credit-bearing program, and all participants are enrolled as visiting summer students through Rice University. They are eligible to receive up to 3 credits through the ELEC 490: Electrical Engineering Research Projects course and, upon conclusion of NanoJapan, can request a transcript from Rice University for possible transfer back to their home university transcript. All students are provided with international health insurance by the NanoJapan Program and are enrolled in Rice University's global assistance program, International SOS. Participants are required to register with the U.S. Department of State through their Safe Traveler Enrollment Program (STEP). Students are also required to rent a Japanese cell phone for the duration of their stay abroad; providing a direct point of contact with students in case of an emergency.

\section{NANOJAPAN PROGRAM HIGHLIGHTS}

\section{Fostering Excellence in Research Collaborations between the U.S. and Japan}

The focus topic of our NSF-PIRE grant is THz science and technology of nanosystems, i.e., studies of the physics and applications of $\mathrm{THz}$ (or $10^{12} \mathrm{~Hz}$ ) dynamics of carriers and phonons in nanostructures and nanomaterials. Some of the ongoing research projects include active and/or plasmonic $\mathrm{THz}$ sources and detectors based on graphene, ${ }^{8-10}$ development of $\mathrm{THz}$ polarizers ${ }^{11,12}$ and detectors ${ }^{13-15}$ based on aligned single-wall carbon nanotubes, ultrafast optical spectroscopy of coherent $\mathrm{THz}$ phonons in carbon nanomaterials, ${ }^{16-18}$ and $3 \mathrm{D}$ micro-fabrication of single-wall carbon nanotube/polymer composites by two-photon polymerization lithography. ${ }^{19}$

The electromagnetic spectrum from 0.1 to $10 \mathrm{THz}$ offers many opportunities to study physical phenomena, with potential payoff in numerous technologies. However, this regime is poorly developed compared to those of electronics $(<100 \mathrm{GHz})$ and photonics $(>10 \mathrm{THz})$. By a judicious combination of $\mathrm{THz}$ technology and nanotechnology, we significantly advance our understanding of THz physics, while improving existing, and developing new, $\mathrm{THz}$ devices. Although Japan and the U.S. are global leaders in both THz research and nanoscience research, there remain obstacles to further collaboration between them, primarily linguistic and cultural barriers. By breaking down these barriers, our NSFPIRE achieves long-term scientific and societal impact, providing future generations of researchers with an understanding of both the culture, and state-of-the-art technology, in each country.

Intellectual Merits: This research (a) advances our quantitative understanding of the THz dynamics of interacting, confined, and driven electrons in nanostructures, (b) grows, synthesizes, and fabricates novel nanostructures for $\mathrm{THz}$ study and applications, (c) advances our cutting-edge experimental techniques in THz spectroscopy and imaging, and (d) provides new knowledge useful for developing novel semiconductor or carbon-based devices that operate in the $\mathrm{THz}$ "technology gap."

Broader Impacts: Our program (a) fosters interest in nanotechnology (freshmen and sophomores), (b) prepares the next generation of graduate students in nanoscience and nanotechnology through on-going engagement with NanoJapan alumni (juniors and seniors), (c) adds to the skill set of active nanoscience researchers (graduate students and faculty), (d) creates participants who are internationally aware and have a specific interest in Japan (all levels), (e) simultaneously educates participants in culture, language, and technology (all levels), and (f) yields transformational institutional change, developing a new model for multidisciplinary collaboration among scholars in engineering, the sciences, and the international education. 
Each NanoJapan student enters into an on-going PIRE international research project team through our international coadvising model (see section 3.2 below). NanoJapan students are assigned to work on a small part of an on-going PIRE research collaboration between their Japanese host lab and the lab of their U.S. co-advisor. Some sample research topics for 2013 NanoJapan students include:

- $\mathrm{THz}$ spectroscopy of large-scale graphene on various substrates

- High-sensitivity room temperature $\mathrm{THz}$ senor based on impact ionization

- Inter-digitated antennas for broadband $\mathrm{THz}$ generation

- Photothermoelectric p-n Junction photodetectors based on macroscopic films of aligned carbon nanotubes in the THz range

Though NanoJapan students spend just eight weeks in their Japanese research lab, they often make significant contributions to the research project during their time in the program. To date, eleven NanoJapan students have been named as authors on peer-reviewed journal articles arising from their NanoJapan research projects in prestigious journals such as Applied Physics Letters, ${ }^{20,21}$ Carbon ${ }^{19}$, Physical Review B, ${ }^{22,23,24}$ and Nano Letters. ${ }^{15}$

\section{International co-Advising Model}

In the first five years (2006-2010), the PIRE PI (Kono) served as the U.S. co-advisor to all NanoJapan students. While initially this helped ensure a solid foundation in the development and mentorship of research projects well-suited to the needs of young undergraduates, our other PIRE researchers in the U.S. expressed interest in working more closely with NanoJapan students. In 2012, we developed the model of pairing each student with both a Japanese host professor and a U.S. co-advisor. The U.S. co-advisor is the PIRE researcher most closely collaborating with the host lab and therefore can provide advisement not only on the research project but also the culture of the host lab. This model strengthens integration into the ongoing PIRE international research projects, allows for more personalized advising, draws on the U.S. researcher's personal experience, and broadens the student's network to include both Japanese and U.S. researchers. While this model is often used with graduate students who may be co-advised by professors from two different institutions, we have found it to be a powerful mechanism for fostering close research collaboration with undergraduate students abroad. Furthermore, this model enables young undergraduates to get a better sense of what being a member of an international team is like and prepares them well for the realities of graduate school and collaborative research.

As J. Meagan Lloyd, a 2012 NanoJapan participant from Carnegie Mellon University who conducted research in the Kawano Lab at Tokyo Institute of Technology, shared at the end of her summer experience, "I am so fortunate to have participated in this research experience and have learned so much this summer, both academically, culturally, socially. It has truly been a privilege to work with these amazing individuals, scientists and engineers."
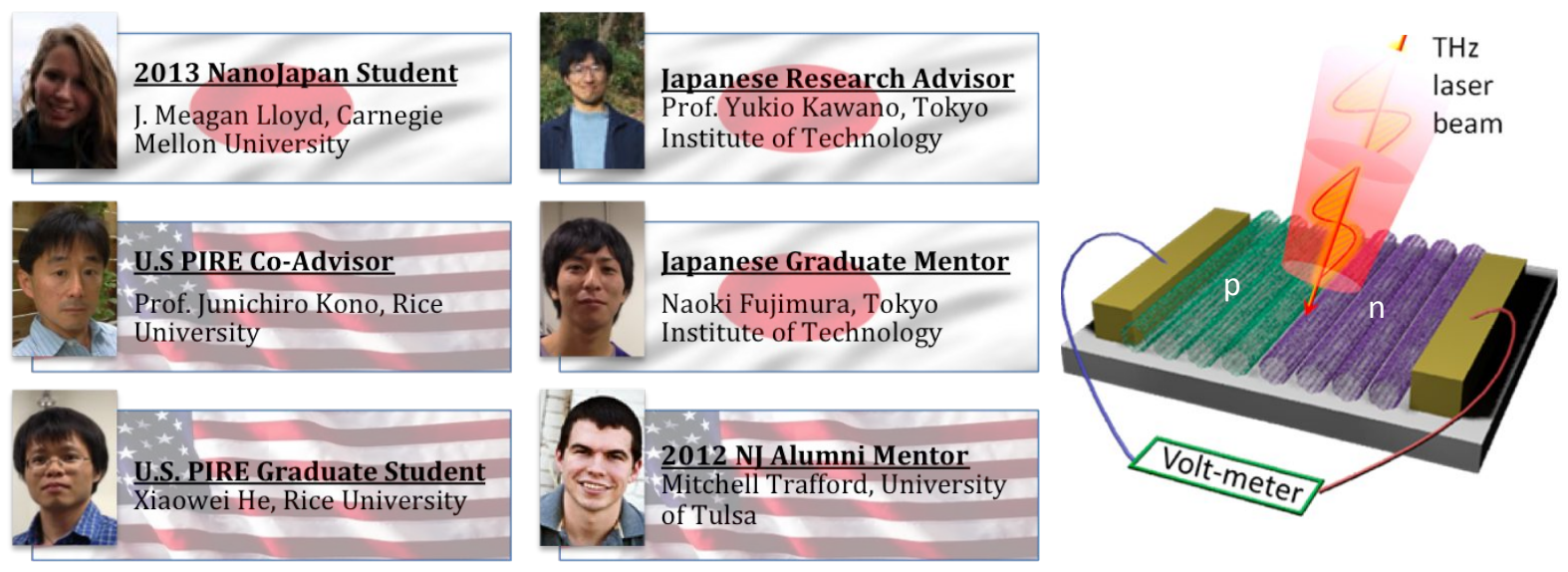

Figure 2. NanoJapan International Co-Advising Model for Prof. Yukio Kawano's lab at the Tokyo Institute of Technology, showing the successful collaboration on the development and characterization of a carbon nanotube THz detector. ${ }^{15}$ 


\section{Weekly Written Reflections}

Throughout the summer, students complete weekly 'blogs' that summarize both progress in their research and cultural adjustment. Each week we ask the students to respond to questions about cultural differences and the role of scientific research in Japan. The formal nature of these assignments, required to receive a grade and credit for the program, ensures that all participants reflect on the nature of their experience in Japan, issues related to cultural adjustment and understanding, gender and career development in nanoscience, and differences between research in the U.S. and Japan.

One alumnus even asked to use the NanoJapan weekly report questions again the following summer when she was participating in another international research experience program in China. As she shared, "I think that the weekly entries we submitted ... allowed us to 'capture the moment' as we experienced things rather than summarizing everything up at the very end. I also think that everyone in our group benefitted a lot $\mathrm{f}[\mathrm{r}] \mathrm{om}$ the questions you posted as prompts, and that they really helped us reflect on our experiences in a way that was meaningful to people who read our entries. So I was thinking it would be helpful to me and the students in Beijing with me to do the same writings this year too."

\section{Fostering U.S.-Japanese Student Engagement}

Each NanoJapan participant is assigned an English-speaking Japanese graduate student or post-doctoral research who is working on the larger overall PIRE research project within that lab to serve as their day-to-day research mentor. The PIRE Japanese student often works with multiple NanoJapan students over the course of their master's or Ph.D. program and benefits from the opportunity to mentor young U.S. undergraduates and strengthen their conversational English skills. Japanese professors report that the U.S. students' enthusiasm and motivation for research positively impacts the lab. As one Japanese professor shared, "[The NanoJapan students] never hesitate to ask questions to our colleagues. Seeing these good attitudes, our Japanese students also get more active, and even change their attitudes for their research in a good way. This is definitely a big benefit for our laboratory."

NanoJapan has also fostered reciprocal opportunities for Japanese students. Since 2006, our collaborating research laboratories in Japan have sent Japanese students to Rice University for short-term internships through the NanoREIS: Research Experiences for International Students program. To date, a total of 72 Japanese students and post-doctoral researcher have participated in NanoREIS, and the program has also enabled an additional 8 students from China, India, and South Korea to conduct short-term research at Rice University in TeraNano PIRE or related S\&E laboratories.

Funding for these short-term research internships must be provided by the international student's home university, and potential interns must submit a research project proposal, in English, outlining their preferred research host lab at Rice University, research topic, and duration of internship. In addition to research, visiting international research interns are also given an introduction to campus life in the U.S. through participation in Rice Graduate Association events and programs sponsored by the Office of International Students and Scholars such as their free conversational English courses. Upon their return to their Japanese home university, NanoREIS alumni are often assigned to be graduate student mentors to the next NanoJapan student placed in their research lab. The NanoREIS experience enables Japanese graduate student mentors to better advise NanoJapan students on some of the differences in research approaches and culture between the U.S. and Japan.

The NanoREIS structure proved invaluable when the March 2011 earthquake and tsunami forced us to reverse the program design and bring 14 U.S. and 25 Japanese students to Rice for the '2011 Reverse NanoJapan' Program. ${ }^{25,26}$ Rice's research facilities were made available to the Japanese students whose research had been suspended due to energy shortages and other after-effects of the disaster in Japan. At the same time, the U.S. students were able to still be involved in international research collaboration with a Japanese student, one of the hallmarks of the NanoJapan Program. The NSF offered supplemental funding through a special allocation for projects impacted by the earthquake and tsunami, which enabled us to offset some travel and program costs for the Japanese student participants. The Office of the President at Rice University also provided full funding for on-campus housing in the graduate apartments, enabling the U.S. and Japanese students to live together for a more robust intercultural experience. In-kind support in the form of guest speakers, special workshops, and other cultural events was also provided by a range of Houston-area organizations including the Japanese Consulate in Houston, the Japan Association of Greater Houston, and Kaminari Taiko. 


\section{Alumni Engagement}

NanoJapan seeks to develop a shared network of peer students with a keen interest in nanoscience research across all program years. A formal part of the program structure is the NanoJapan Alumni Mentor Program. Once selected into the program, each current-year NanoJapan participant is matched with an alumnus from the year prior, typically the student who conducted research in the same host lab that the current participant has been placed into. Mentors engage with the current-year students prior to departure regarding what to expect in their host research lab and city in Japan. This program also gives the alumnus an opportunity to reflect back on their NanoJapan experience and better understand how to best convey the impact of the program to the next generation of participants.

The intergenerational NanoJapan Alumni Network has proven particularly helpful when students are considering future graduate schools or program. Among NanoJapan alumni who have graduated to date, $65 \%$ are pursuing or have received a graduate degree in a science or engineering field from 33 different U.S. and international graduate institutions. Alumni also have a strong track record with major fellowships including 11 NSF Graduate Research Fellowships, 2 National Defense Science and Engineering Fellowships, 1 Hertz Fellowship, 4 Goldwater Scholarships, and 1 Churchill Scholarship. Informal NanoJapan alumni groups have developed among graduate students at universities such as Stanford, with 7 alumni, and the Massachusetts Institute of Technology, with 8 alumni.

International engagement among NanoJapan alumni also remains high with $23 \%$ of alumni pursuing other international opportunities including semester study abroad, summer research abroad, and graduate study abroad. Two alumni have participated in the University of Michigan's Optics in the City of Light IREU program in France and two alumni participated in Clarkson University's Advanced Materials for a Sustainable Development International Research Experience for Students program at Tsinghua University in China. NanoJapan alumni have also pursued graduate study abroad with international fellowship support at Australia National University, Hong Kong University, the King Abdullah University of Science and Technology, and the University of Cambridge. Preeya Kuray, a NanoJapan 2012 participant from Rutgers University who conducted research in Prof. Kawta's lab at Osaka University, was recently selected to participate in the highly competitive Japan English Teaching (JET) program and will be spending the 2014-2015 academic year teaching English in Yamaguchi Prefecture.

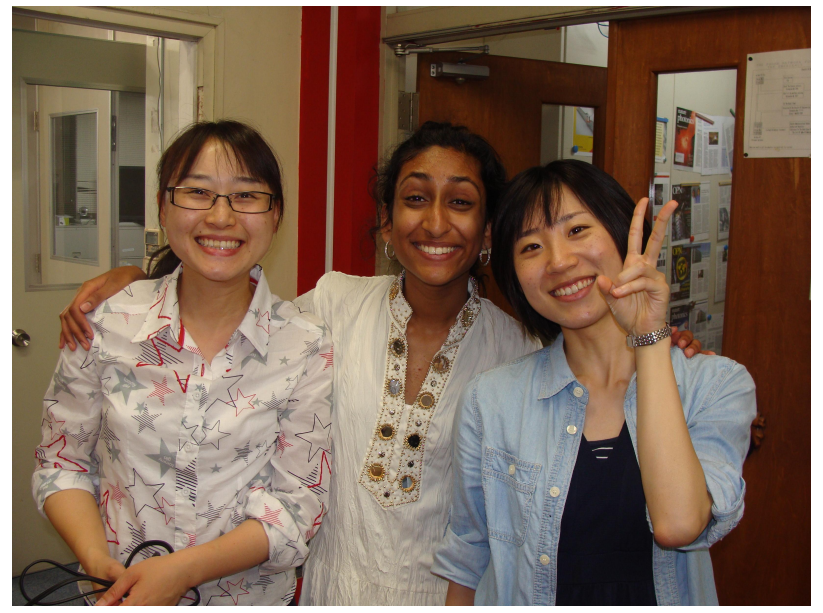

Figure 3. Preeya Kuray with Kyoko Masui (left) and Kozue Watanabe (right) in Prof. Satoshi Kawata's lab at Osaka University during the 2012 NanoJapan Program. As she recently shared, "I am pursuing JET for both cultural and personal enrichment. I hope to tie Japanese into my future career in the sciences and after JET I will be better equipped to do so. NanoJapan fostered my interest in Japanese culture and language. It not only provided me with a fruitful research experience to jumpstart my career, but also provided me with cultural enrichment that sparked my interest in pursuing Japanese studies. I hope to utilize the skills I have learned during NanoJapan to make a meaningful impact on my students during the JET program." 


\section{NanoJapan Follow-on Project}

All NanoJapan students are required to carry out a follow-on project at their home university or in their local/home community in the academic year following participation in the program. Follow-on Projects are student designed and initiated and seek to encourage other students to pursue study or research in science or engineering, especially in emerging areas such as $\mathrm{THz}$ science and technology, and/or pursue international research and study opportunities at the undergraduate or graduate level. Students are strongly encouraged to develop projects to be implemented in local middle schools or high schools, fostering engagement with the next generation of science and engineering undergraduates.

\section{PROGRAM ASSSESSMENT}

The program outcomes are assessed using a combination of quantitative and qualitative methods on the following dimensions: student attitudes towards the engineering profession; Japanese language proficiency; intercultural sensitivity and competency; and career choice. In assessment of program outcomes, students identified three major themes regarding the impact of NanoJapan: a) enhanced confidence as a researcher, b) an introduction to and better understanding of what graduate study and research is like, and c) the development of a professional network of U.S. and Japanese researchers and student peers. ${ }^{9}$

\section{Attitudes}

All participants complete PIRE Participant Questionnaires, an assessment tool developed by the NSF to measure outcomes for all PIRE programs. The PIRE Questionnaire specifically measures participants' attitudes towards international research and the perceived impact of the PIRE project on their career goals. Of the 76 students who completed the PIRE Questionnaire, $80 \%$ strongly agreed that the international dimension of the research project was an important factor in my decision to join the project; $90 \%$ strongly agreed that foreign collaborators can provide valuable contributions to US science projects; and $82 \%$ agreed that the cultural context of science has an impact on how the research is conducted. The responses by NanoJapan participants no doubt reflect a strong selection bias - the students who apply and are selected for the program do so because they desired to participate in an international research experience. The PIRE Participant Questionnaire should not be considered a measure of the impact of the NanoJapan Program. Rather, it demonstrates that an international dimension to a research experience can be a very important factor in recruiting students to participate in scientific research.

\section{Language Proficiency}

The students' progress with Japanese language is assessed using the Oral Proficiency Interview (OPI), a valid and reliable measure for assessing how well a person speaks a language. A trained rater, who completes a 20-30 minute faceto-face or telephonic interview with the examinee, administers the OPI. The interview is interactive and continuously adapts to the interests and abilities of the speaker. The speaker's performance is compared to the criteria outlined in the American Council for Teachers of Foreign Languages (ACTFL) Proficiency Guidelines - Speaking. We administered the OPI as a post-test to all NanoJapan students and a pre-test to any student who had previously studied or learned Japanese. The chart below summarizes the results for the 83 NanoJapan students who have not previously studied Japanese.

Among the 80 students with no previous Japanese language experience, $46 \%$ were evaluated on the Oral Proficiency Interview at a Novice Mid (roughly equivalent to 150 instructional hours) and $31 \%$ at a Novice High (roughly equivalent to 270-300 instructional hours) by the end of the summer.

This is significant since the students complete only 45 hours of formal instruction during the 3 -week orientation program. During the 8-week research internship students who wish to continue with Japanese language study do so informally via language classes taught by volunteer teachers, informal language exchanges with Japanese members of their research group, or self-study utilizing online resources recommended by NanoJapan language teachers. These results show that NanoJapan participants are highly motivated language learners and continue to utilize and study the language outside of formal language classes. 


\begin{tabular}{|l|l|}
\hline Rating & Students $(\mathbf{n}=\mathbf{8 3})$ \\
\hline Novice Low & 10 \\
\hline Novice Mid & 39 \\
\hline Novice High & 26 \\
\hline Intermediate Low & 6 \\
\hline Intermediate High & 2 \\
\hline
\end{tabular}

Figure 4. Japanese Oral Proficiency Interview: Post-test Results from participants with no prior Japanese language study or experience prior to participation in NanoJapan.

Research on the gains by STEM majors studying a foreign language is scarce, and our analysis of the reasons for the impressive progress by NanoJapan students is anecdotal. Students are immersed among Japanese speakers and many students describe themselves as highly motivated to learn Japanese. One study that assessed features of study abroad programs that affected student language learning, also concluded that in programs with orientations that included a cultural component, as does NanoJapan, students demonstrated greater improvement in their oral proficiency at the end of study abroad. ${ }^{6}$

\section{Intercultural Competency}

We utilize two instruments to assess intercultural competency, the Intercultural Development Inventory and the Engineering Global Preparedness Index. The Intercultural Development Inventory (IDI) is theoretically grounded in Milton Bennett's "Developmental Model of Intercultural Sensitivity," a frequently-cited developmental model that identifies six progressive stages through which individuals pass in adapting interculturally. The results of the IDI place an individual at a point along this six-stage developmental continuum, from ethnocentric to ethnorelative. The stages of the DMIS are denial, defense, minimization, acceptance, adaptation, and integration. ${ }^{27}$ While the IDI does not measure learning specific to Japan, it does provide reliable and valid measure of an individual's ability to operate effectively within a different cultural context. We administered the IDI as a pre and post-assessment to all NanoJapan students. We did not find significant changes along the IDI's developmental continuum in the scores of the NanoJapan participants, which may be due to the short duration of the NanoJapan program. Interestingly, a similar research program based in Asia, the Pacific Rim Experiences for Undergraduates (PRIME) project, reported nearly identical IDI results for participants in their summer research program. ${ }^{28}$

We introduced a new measure in 2013 to assess global preparedness, the Engineering Global Preparedness Index (EGPI), a multi-dimensional engineering global preparedness index that is theoretically grounded in the global citizenry theory recommended by Zeichner, ${ }^{29}$ Ragusa, ${ }^{30}$, and Banks. ${ }^{31}$ It is designed to directly measure engineering students' preparedness to work in global workforces. There are total of 30 items on the EGPI, with 3-4 items per subscale (totalling 18 items) and demographic questions. The four subscales in the EGPI directly align to important "soft skills" needed both by engineers and other globally prepared professionals. ${ }^{32}$

The 2013 NanoJapan student participants increased slightly in overall engineering global preparedness and on three of the four EGPI subscales: Engineering Community Connectedness, which measures 'global kinship,' Global Engineering Efficacy, which measures the extent to which a person believes he or she can make a difference, Engineering Ethics and Humanitarian Values, or a concern for other people in the world. Interestingly, the NanoJapan students demonstrated a decline on the measure of Engineering Global Centrism, which may reflect that our program has not asked students to consider their research in context of global needs.

It is important to bear in mind that the pre-test scores for the NanoJapan students are, as compared with other US undergraduate students, quite high. ${ }^{33}$ We know that many students apply to NanoJapan because they are interested in international experiences, so it is perhaps not surprising that students begin the program scoring high on a measure of global preparedness. However, the NanoJapan students indicate important changes at the conclusion of their internship in their global preparedness which suggests that the experience abroad itself impacts students on these dimensions. 


\section{Attitudes and Self-Efficacy}

Our final assessment instrument is the George Tech International Internship Survey, which we also administered as a pre and post assessment to students in Summers 2012 and 2013. The instrument is divided into three sections. In the first section of the instrument, students rate items related to knowledge, abilities, and skills on two measures: i) how important they consider the item (on a scale from very important to not important) and ii) their assessment of their relative preparation (on a scale from very well prepared to not prepared). In the second section, students are asked to assess their confidence in their workplace skills and abilities. The final section asks students about their career plans. The instrument was developed by the Georgia Tech Office of Assessment and uses an externally validated General SelfEfficacy Scale to assess an individual's ability to cope with stressful life events. ${ }^{34}$

NanoJapan students scored the importance of most of the items on the Georgia Tech Internship Survey as higher on the post-test as compared with the pre-test. The items for which students reported the largest changes in terms of importance included:

- an ability to communicate orally and in writing;

- exercise of leadership skills;

- working effectively as part of a multi-disciplinary or cross-functional team;

- effectively resolving interpersonal conflict;

- effectively working in the host country's culture;

- and effectively practicing their discipline in a different social or cultural setting.

The NanoJapan students reported on the post-test that they were more prepared to:

- communicate orally and in writing;

- exercise leadership skills;

- effectively resolve interpersonal conflict;

- communicate in their host country's language in a social or professional setting;

- work effectively and efficiently in a cross-cultural environment;

- and approach problems from different perspectives.

The NanoJapan students rated all items related to technical or research abilities as slightly more important on the posttest. However, they also reported significant gains in their assessment of their preparation to analyze and interpret data; think critically and logically; carry out projects independently; identify, formulate, and solve problems within their discipline context; and design a system, component, or process to meet a desired need and quality.

Interestingly, the NanoJapan students rated their perceived importance of the following items lower on the post-test as compared with the pre-test: conversational fluency, professional or technical fluency, and an ability to professionally collaborate with persons in their host country's workplace environment. This may reflect that when the assessment was completed as a pre-test, the students had not yet experienced living and working in Japan and were nervous about their own lack of Japanese language skills or abilities to work in the Japanese labs. By the end of the summer, they assessed these items as less important because they had themselves survived without fluency in Japanese and had still learned to collaborate with their research host laboratories.

\section{Impact on Career Choice and Long-Term Goals}

Perhaps our most compelling outcomes data is with regard to post-program activities. A goal for the program is to encourage students to pursue graduate study in the physical sciences. Among program alumni, $72 \%$ indicated at completion of the program they were likely to pursue a career in science and engineering, a percentage that tracks closely with actual program results. In the fall of 2012, we conducted a qualitative program assessment on long-term impact of the program with all alumni from 2006 to 2011, a total of 94 students. Thirty students, or 31.9\%, of alumni surveyed responded. We will conduct a follow-on program assessment in 2015 to include all program participants. The students reported the following themes when asked about the impact of the program on their career goals: 
Enhanced confidence as a researcher: Students reported that the experience of conducting nanotechnology research improved their self-confidence working in a lab. Students also reported that the experience of successfully living independently in another country in which they did not speak the language made them more confident in general. In one typical response, a NanoJapan alum reported, "The fact that I have had the experience of relocating to a different culture and lab environment and successfully adapting to it means that I have greatly increased comfort and confidence in research environments. After all, relocating to a different lab in the U.S. will always pale in comparison to relocating to a lab on the other side of the world."

Training for graduate school: Several students reported that the NanoJapan experience was important because it provided a first exposure to the science. For example, one alum wrote, "Without realizing it at the time, NanoJapan served as one of the most transformative experiences in my life - the introduction to magnetism and spin-related research that eventually led me to pursue a $\mathrm{PhD}$ in magnetics." An even more frequent response was that the experience of conducting research was itself a valuable preparation for the realities of graduate school. One alum explained, "Looking back now, I see that my lab in NanoJapan gave me a realistic taste of graduate school life (the good AND the bad) that many students lack when they apply for graduate school. I know more than a few people that have left their graduate programs because research was not what they expected. Many of these people had performed research during their undergraduate studies as well, which speaks to the accuracy of the NanoJapan experience in particular."

Professional network: The final theme that emerged from these qualitative responses was the importance of building a professional network. Some program alums explained that they were still in contact with their Japanese research hosts. Others described that the NanoJapan program itself provided them with a valuable network of peers interested in science, with whom they could consult about graduate school and career options.

\section{PROGRAM IMPACT AND CONCLUSION}

The NanoJapan Program has received national recognition as a model for the expansion of international opportunities for science and engineering students. In 2008, the NanoJapan Program was awarded an Institute of International Education Heiskell Award as a 'Best Practice in Study Abroad' for expanding international opportunities for engineering and science students. ${ }^{35}$ In 2013, the NanoJapan Program was included in a National Academy of Engineering report profiling 29 programs that "Infused Real World Experience into Engineering Education". The report highlighted these program as national models for encouraging enhanced richness and relevance of the undergraduate engineering education experience" that would "produce better prepared and more globally competitive graduates". ${ }^{36}$ Beyond these national awards, as a program that recruits students from universities nationwide, NanoJapan also serves as a research catalyst for students who may have limited research opportunities at their home universities; particularly in the emerging area of $\mathrm{THz}$ studies in nanosystems.

The assessment measures used for the program suggest that students perceive that NanoJapan enhances their understanding of international research. More importantly, we know that the international dimension of the program is a major influence on students' decision to apply to the program in the first place, and thereby get exposure to nanoscale science research while early in their academic career. Students report that the experience of living independently in Japan enhances their self-confidence, and that the independent research project is perceived as a kind of 'training ground' for the realities of graduate school. This suggests that the NanoJapan Program has proven to be a successful model for the expansion of international opportunities for science and engineering students and contributes to the cultivation of the next generation of U.S. graduate students in the physical sciences. As one NanoJapan alumnus shared:

"NanoJapan is scientific discovery, world discovery, and self-discovery all in one summer. It gives young undergraduates the chance to experience graduate student life in a state of the art lab on the other side of the world...It is the opportunity to be part of something much bigger than an individual both in respect to being involved with a group of like-minded individuals and to contribute to the world of science that transcends cultural and language barriers."

We would like to acknowledge the financial support of the National Science Foundation through grant nos. OISE0530220 and OISE-0968405 as well as the contributions of the TeraNano PIRE team members to the NanoJapan Program, including Keiko Packard, Mitsuaki Shimojo, Jonathan Bird, and Christopher Stanton. 


\section{REFERENCES}

[1] Institute of International Education, "Fields of study of U.S. study abroad students, 2000/01-2010/11," Open Doors Report on International Educational Exchange, 2013, http://www.iie.org/Research-and-Publications/OpenDoors/Data/US-Study-Abroad/Fields-of-Study (15 July 2014).

[2] National Science Foundation. "Empowering the nation through discovery and innovation," NSF Strategic Plan for Fiscal Years $2011-2016$ NSF 11-047, 8 (2011).

[3] Institute of International Education, "International students by field of study, 2011/2012 - 2012/13," Open Doors Report on International Educational Exchange, 2013, http://www.iie.org/Research-and-Publications/OpenDoors/Data/International-Students/Fields-of-Study (15 July 2014).

[4] Parkinson, A., "Engineering study abroad programs: formats, challenges, and best practices," Online J. Eng. Edu. 2, 2 (2007).

[5] National Science Foundation WebCASPAR Database: IPEDS Completion Survey. "Fraction of Bachelor's Degrees Earned by Women, by Major," http://www.aps.org/programs/education/statistics/womenmajors.cfm (15 July 2014).

[6] Vandeberg, M., "Intervening in student learning abroad: a research based inquiry," Int. Edu. 20(1) 15-27, (2009).

[7] The Forum on Education Abroad, "Standards of good practice for short-term education abroad programs," (2009).

[8] Ryzhii, V., Ryzhii, M., Mitin, V., Shur, M.S., Satou, A., and Otsuji, T., "Terahertz photomixing using plasma resonances in double-graphene layer structures," J. Appl. Phys. 113, 174506 (2013).

[9] Ryzhii, V., Otsuji, T., Ryabova, N., Ryzhii, M., Mitin, V. and Karasik, V., "Concept of infrared photodetector based on graphene-graphene nanoribbon structure," Infrared Phys. Technol. 59, 137-141 (2013).

[10] Ryzhii, V., Otsuji, T., Ryzhii, M., Ryabova, N., Yurchenko, S. O., Mitin, V., and Shur, M. S., "Graphene terahertz uncooled bolometers", J. Phys. D: Appl. Phys. 46, 065102 (2013).

[11] Ren, L., Pint, C. L., Booshehri, L. G., Rice, W. D., Wang, X., Hilton, D. J., Takeya, K., Kawayama, I., Tonouchi, M., Hauge, R. H., and Kono, J., "Carbon nanotube terahertz polarizer," Nano Lett. 9, 2610-2613 (2009).

[12] Ren, L., Pint, C. L, Arikawa, T., Takeya, K., Kawayama, I., Tonouchi, M., Hauge, R. H., and Kono, J., "Broadband terahertz polarizers with ideal performance based on aligned carbon nanotube stacks," Nano Lett. 12, 787-790 (2012).

[13] Nanot, S., Cummings, A. W., Pint, C. L., Ikeuchi, A., Akiho, T., Sueoka, K., Hauge, R. H., Léonard, F., and Kono, J., "Broadband, polarization-sensitive photodetector based on optically-thick films of macroscopically long, dense, and aligned carbone= nanotubes," Sci. Rep. 3, 1335 (2013).

[14] He, X., Nanot, S., Wang, X., Hauge, R. H., Kane, A. A., Goldsmith, J. E. M., Léonard, F., and Kono, J., "Photothermoelectric p-n junction photodetectors with intrinsic polarimetry based on macroscopic carbon nanotube films," ACS Nano 7, 7271-7277 (2013).

[15]He, X., Fujimura, N., Lloyd, J. M., Erickson, K. J., Talin, A. A., Zhang, Q., Gao, W., Jiang, Q., Kawano, Y., Hauge, R. H., Léonard, F., and Kono, J., "Carbon nanotube terahertz detector," Nano Lett. 14, 3953-3958 (2014).

[16] Sanders, G. D., Stanton, C. J., Kim, J.-H., Yee, K.-J., Lim, Y.-S., Hároz, E. H., Booshehri, L. G., Kono, J. and Saito, R., "Resonant coherent phonon spectroscopy of single-walled carbon nanotubes," Phys. Rev. B 79, 205434 (2009).

[17] Kim, J.-H., Nugraha, A. R. T., Booshehri, L. G., Hároz, E. H., Sato, K., Sanders, G. D., Yee, K.-J., Lim, Y.-S., Stanton, C. J., Saito, R. and Kono, J., "Coherent phonons in carbon nanotubes and graphene," Chem. Phys. 413, 5580 (2013).

[18] Lim, Y.-S., Nugraha, A. R. T., Cho, S.-J., Noh, M.-Y., Yoon, E.-J., Liu, H., Kim, J.-H., Telg, H., Hároz, E., H., Sanders, G. D., Baik, S.-H., Kataura, H., Doorn, S. K., Stanton, C. J., Saito, R., Kono, J., and Joo, T., "Ultrafast generations of fundamental and multiple-order phonon excitations in highly-enriched $(6,5)$ single-wall carbon nanotubes," Nano Lett. 14, 1426-1432 (2014).

[19] Ushiba, S., Shoji, S., Masui, K., Kuray, P., Kono, J., and Kawata, S., "3D micro-fabrication of single-wall carbon nanotube/polymer composites by two-photon polymerization lithography," Carbon 59, 283-288 (2013).

[20] Cooper, L., Amano, H., Hiraide, M., Houkyou, S., Jang, I. Y., Kim, Y. J., Muramatsu, H., Kim, J.-H., Hayashi, T., Kim, Y. A., Endo, M., and Dresselhaus, M. S., "Freestanding, bendable, thin film for supercapacitors using DNAdispersed double walled carbon nanotubes," Appl. Phys. Lett. 95, 233104 (2009).

[21] Yuan, H. T., Toh, M., Morimoto, K., Tan, W., Wei, F., Shimotani, H., Kloc, Ch., and Iwasa, Y., "Liquid-gaged electric-double-layer transistor on layered metal dichalcogenide, $\mathrm{SnS}_{2}$," Appl. Phys. Lett. 98, 012102 (2011).

[22] Murakami, Y., Lu, B., Kazaoui, S., Minami, N., Okubo, T., and Maruyama, S., "Photoluminescence sidebands of carbon nanotubes below the bright singlet exitonic levels," Phys. Rev. B, 79, 195407 (2009). 
[23] Ren, L., Zhang, Q., Pint, C. L., Wójcik, A. K., Bunney, M., Arikawa, T., Kawayama, I., Tonouchi, M., Hauge, R. H., Belyanin, A., and Kono, J., "Collective antenna effects in terahertz and infrared respose of highly aligned carbon nanotube arrays," Phys. Rev. B 87, 161401(R) (2013).

[24] Nugraha, A. R. T., Rosenthal, E. I., Hasdeo, E. H., Sanders, G. D., Stanton, C. J., Dresselhaus, M. S., and Saito, R., "Excitonic effects on coherent phonon dynamics in single-wall carbon nanotubes," Phys. Rev. B, 88, 075440 (2013).

[25] Boyd, J., “Tsunami’s impact warrants location change for summer study abroad,” Rice News, May 11, 2011. http://news.rice.edu/2011/05/11/reverse-nanojapan-rice-to-host-25-30-japanese-students-2/ (16 July 2014).

[26] Photonics Spectra staff, "Earthquake impact reverses NanoJapan plans," Photonics Spectra, 1 August 2011, http://www.photonics.com/Article.aspx?AID=47964 (16 July 2014).

[27] Hammer, M.R., Bennett, M.J, and Wiseman, R., "Measuring intercultural sensitivity: The intercultural development inventory," Int. J. of Intercultural Relations, 27(4), 421-433 (2003).

[28] Arzberger, P., Wienhausen, G., Abramson, D., Galvin, J., Date, S., Lin, F.-P., Nan, K. and Shimojo, S., "PRIME: An integrated and sustainable undergraduate international research program," Adv. Eng. Edu. 2, 2-xxx (2010).

[29]Zeichner, K., [Teacher Education and the Struggle for Social Justice], Routledge, New York (2009).

[30] Ragusa, G., "Engineering preparedness for global workforces: Curricular connections and experiential impacts," Proc. Am. Scty. of Eng. Ed. (2011).

[31] Banks, J., "Teaching for social justice, diversity, and citizenship in a global world," The Educational Forum, 68, $296-305$, (2004).

[32] Ragusa, G., "Engineering global preparedness: parallel pedagogies, experientially focused instructional practice," Int. J. Eng. Edu. 30(2), 1-12 (2014).

[33] Matherly, C. A., Phillips, S. R., and Kono, J., "Why invest in international research experiences for undergraduates?: Intercultural maturity in domestic and international REU participants," Am. Scty. of Eng. Ed. Int'l Forum, Indianapolis, IN, June 14, 2014, http://www.asee.org/conferences-and-events/conferences/internationalforum/2014/11040.pdf (22 July 2014).

[34] Gordon, J. "Georgia Institute of Technology: Assessing global learning, lessons from the field." NAFSA: Assoc. of Int'l Educators, Washington, DC (2013).

[35] Institute of International Education, "2008 Heiskell Award winners: study abroad," 2008, http://www.iie.org/WhoWe-Are/IIENetwork-Membership/Heiskell-Awards/Study-Abroad/2008-Rice-Tulsa (16 July 2014).

[36] "Infusing Real World Experience Into Engineering Education", National Academy of Engineering, pps. 2 and 33 (2013). 